\section{MS12-03 The ins-and-out of a membrane protein; Structure-function through alternative crystallisation and harvesting approaches}

Anna Polyakova ${ }^{1}$, Scott Jackson ${ }^{2}$, Alexander Cameron ${ }^{3}$, Peter J.F. Henderson ${ }^{1}$, Arwen R. Pearson ${ }^{4}$

1. Astbury Centre for Structural Molecular Biology, University of Leeds, Woodhouse Lane, Leeds, LS2 9JT, United Kingdom

2. Institute of Molecular Biology and Biophysics, ETH Zurich, HPK G9.3, Otto-Stern-Weg 5, Zurich, 8093, Switzerland

3. Department of Life Sciences, University of Warwick, Gibbet Hill Road, Coventry, CV4 7AL, United Kingdom

4. Hamburg Centre for Ultrafast Imaging, Universität Hamburg, Luruper Chaussee 149, Hamburg, 22761, Germany

email: cm10ap@leeds.ac.uk

Secondary-active trans-membrane transporters couple the 'downhill' movement of ions with the 'uphill' movement of essential substrates, such as neurotransmitters or metabolites, across the cell membrane. Disruption of these vital processes is linked to some severe diseases in humans, for example Parkinson's disease.The aim of this project is to elucidate the structural basis of the transport mechanism of secondary-active transporters, using the bacterial sodium-hydantoin transporter Mhp1 as a model system. Currently, a number of static crystal structures of Mhp1 are available, representing snapshots of intermediate states along its transport cycle. However, they provide only limited insights into a highly dynamic process. In order to bridge the gaps between these snapshots, we are using a combination of X-ray crystallography and small-angle X-ray scattering (SAXS). We are using mainly X-ray crystallography to decipher the roles of individual residues in the transport mechanism and their interactions with ligands. A wide variety of strategies have been applied in the crystallisation, harvesting and cryo-protection steps to improve the data obtained from Mhp1 crystals. These include for example modifications to the crystal drop morphology (see figure) to improve crystal size and quality, as well as on-line crystal dehydration and contact-less harvesting by photoablation to minimise damage from cryo-protection and harvesting. In these studies a shrinking in the crystal a- and b-axes (which are parallel to the putative membrane normal) has been observed, giving less anisotropic data, improved electron density maps and has enabled us to solve crystal structures of Mhp1 mutants in complex with various ligands. Moreover, we are using SAXS to obtain information about the overall shape and detergent organisation around the protein in order to gain insight into crystallisation propensity and detergent packing in membrane protein crystals. We are overcoming the challenges imposed by the presence of the detergent molecules by combining SAXS with size-exclusion chromatography. The main goal of this project is to reveal structural and dynamic information that has not been observed before: what routes the substrates take through the protein; what conformational changes occur during substrate binding and release; how is the transport of ions and substrates coupled?
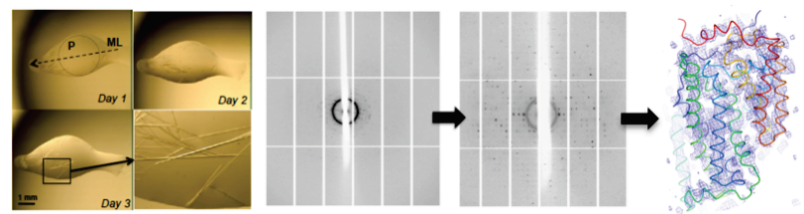

Figure 1. Illustration of how changing the drop morphology has improved crystal size and quality, enabling us to solve crystals of Mhp1 mutants in complex with various ligands.

Keywords: Membrane protein, crystallization 